\title{
The Times They Are A-Changin': Mobile PIM is leaving the Paper Trail Behind
}

\author{
Juha Leino \\ Sanna Finnberg \\ Kari-Jouko Räihä \\ Unit for Computer-Human Interaction School of Information Sciences \\ FIN-33014 University of Tampere \\ juha.leino@gmail.com \\ sanna.finnberg@cs.uta.fi \\ kari-jouko.raiha@cs.uta.fi
}

\begin{abstract}
Use practices in personal information management (PIM) evolve continually. The data from our two studies, a survey study $(n=61)$ on to-do use practices and a diary/interview study $(n=11)$ on smartphone use in PIM, suggests that electronic PIM has finally reached paper-based PIM in popularity. Mobile phones and their development have been integral to this development: Better input possibilities and better screens together with improved Internet accessibility have made it possible for us to increasingly organize our lives with mobiles. Thus, mobiles have to be in the epicenter when designing the PIM applications of tomorrow.
\end{abstract}

Personal information management, PIM, mobile devices, reminders.

\section{INTRODUCTION}

Personal information management (PIM) is "intended to support the activities we, as individuals, perform to order our daily lives through the acquisition, organization, maintenance, retrieval, and sharing of information." [20]. Core tools that support PIM include to-do lists and calendars [11] in addition to email $[2,22]$. They and other PIM tools are available in several media, including paper, PCs, and mobile devices (e.g. mobile phones and PDAs).

Many studies (e.g. $[4,9,15,21]$ ) have shown that paper is the rule and electronic devices and applications more of an exception in PIM. The situation, however, appears to be changing with increasingly smart mobile phones becoming more common. As part of an ongoing project that is developing a widget-based environment for PIM and PIM-related sharing-many PIM activities actually involve other people at many levels [13] we conducted two current PIM practice studies that both showed a growing tendency for using electronic PIM tools: Both for calendars and to-do lists, our participants had moved towards using more electronic than paper-based tools.

Our first study was a survey study of to-do use and of the features respondents saw as most important in to-do applications $(n=61)$. While paper to-dos were still more common overall, the use of electronic todos was clearly catching up when compared to the results of previous studies. In fact, most respondents used both electronic and paper tools for to-dos. Interestingly, mobile devices were widely used for to-dos: Nearly $60 \%$ of the respondents who used electronic PIM mentioned using mobile devices, typically mobile phones, in PIM.
Our second study was a diary/interview study of 11 smartphone users' PIM habits. As lead user practices anticipate those of others, they offer us insight into how things are likely to be in the future [12]. The study showed that among lead users electronic calendars were more popular than paper ones and that to-dos and information scraps were also increasingly done with the mobile. The second study also underlined that it was the advancements in the phones rather than in PIM applications that had made this development possible: It was bigger screens with higher resolutions, better text entry possibilities with QWERTY keyboards, and Internet connectivity that had allowed the participants to start using mobiles in PIM more widely.

Thus, these two studies suggest that with smartphones becoming more commonplace, electronic tools are increasingly used in PIM in the future, and that PIM applications have to take mobility into consideration-the time of PC-only PIM applications is a thing of the past. Users will want to have access to their personal information also on the move, and so being able to access the same personal information with different devices is essential.

However, as mobile devices are more used to browse and use personal information rather than to create and organize it, and as the to-dos and information scraps used when working by the computer are still often stored in the computer, PCs still play an important role. Depending on the device used to access personal information, the application interface has to support users somewhat differently: The interfaces of the mobile devices should focus 
on enabling users to use the existing information and make quick changes to it (crossing things over or ticking them as done) and to make quick entries (short emails or Facebook status updates) while PC interfaces should make it easy to enter and organize large amounts of information in addition to using it.

\section{RELATED WORK}

Most studies thus far have concluded that paperbased PIM is more popular than electronic PIM. The only exception we found is a study of Microsoft workers who used Microsoft Outlook for work [6]. A small majority-59 per cent-used electronic calendars as their primary personal calendar. The reason seemed to be at least partially that since they had to use the electronic calendar at work, they preferred to use it for personal matters as well to avoid having two calendars [6]. Otherwise, the only other study where users used electronic calendars more than paper ones was one where they were forced to do so-and even then, some made printouts for actual use [5].

Earlier findings also show that even when users had portable electronic devices, such as smartphones and PDAs, they commonly ended up using paper [9, $15,21]$. Our smartphone user study suggests that this is now changing. The reasons for this appear to center around the devices having better screens (bigger sizes and better resolutions) and goodenough input facilities rather than better applications, as using catch-alls or co-opting applications is common, as also observed by Bernstein et al. [4].

There are numerous reasons why paper has been so popular. While entering information into electronic devices has been seen as cumbersome [1, 3, 4, 9]-for example, Lin, Lutters and Kim [15] found that 11 out of their 12 participants who owned a PDA still used paper-paper allows free-form entry and free organizing and juxtaposing of information [19]. Furthermore, paper makes it easy to create structure, emphasis, categories and grouping with colors, arrows, and circling in addition to allowing sketching [4, 18]. Moreover, unlike many PIM applications, paper allows entering content at any level of completeness without any application/toolimposed structure $[3,4,6,9,19,21]$.

Unlike electronic devices, paper does not need any start-up time and it never runs out of battery [1] in addition to being almost universally available, as users grab any piece of paper and use it [15]. Furthermore, paper is compact and easy to carry and use [9, 19, 21] —in effect, a catch-all, all-in-one tool par excellence [21].
Such paper tools as calendars offer easy browsing [6] with visual clues as to where in time the user is, and so few people make such mistakes as marking things to a wrong month on a paper calendar while this is a constant problem with electronic ones where information is more abstract and out of the context of the whole [5]. While paper still has the upper hand, the bigger screens and resolutions of such phones as iPhone, N97 and N900 are making using mobile devices in PIM increasingly easier in this regard.

Taylor and Swan [19] sum up the advantages of paper as freeform entry (whatever), freeform organization and system (however), portability (wherever), and accessibility (whoever). While mobile devices cannot match these advantages, they are inching closer, and with other advantages they offer, the better mobile phones of today are increasingly used in PIM and are in fact becoming epicenters of PIM for lead users, as our smartphone study shows.

\section{METHOD}

Our first study was arranged as an online survey in May 2009. The respondents were first asked about their to-do use practices both with multiple choice and open-ended questions and then asked to evaluate the importance of 67 to-do features for themselves with a seven-point scale (from "very important" to "not important at all"). The respondents were able to comment each evaluation in a text field. Respondents were recruited by advertising the survey in the mailing lists of our university. Four movie tickets were raffled among the respondents.

The survey was given in English and it was completed by 61 respondents, of whom 35 were females and 26 males, 45 Finns and 16 from other nationalities (mostly from Europe and North America), as the mailing lists also included exchange students. The results for Finns and non-Finns were similar, and thus will not be discussed separately. Most were university students and relatively young: 45 were 20-29 years of age, 10 were $30-39,3$ were $40-49$, and 2 were $50-59$. One did not provide his age.

To gain more in-depth information on the use practices, the survey study was followed with a second study. Eleven smartphone users were first asked to keep a diary of how they used their phones to help them remember things and manage their lives for five consecutive days (they were given the choice of using either electronic or paper diary). They were then interviewed individually about how they used their phones for PIM. In addition, they filled out an online background questionnaire. The data was collected in Nov and Dec, 2009. 
The participants were given a ready-made diary form for filling in for each use instance a description, time, application used, context (home/work/other), and whether the matter was completed successfully. The semi-structured interviews, conducted after the diaries were completed, lasted 30-60 minutes per participant.

Eight participants were recruited from an introductory class to interactive technology and two were recruited through personal networks. In addition, we included the pilot study participant-a fellow researcher not involved in the project-as the 11th participant since the pilot study did not bring any significant changes to the study setting. The prerequisite for recruitment was owning and using a smartphone. The participants were aged 20-39 and 9 were students with 5 majoring in computer science.

In interpreting our results we must keep in mind that our 61 respondents, mainly university students, allow only limited generalizing of results. The same limitation applies to the smartphone user study with 11 participants who were mainly students. Thus, the results more represent the practices of the up and coming generations of better-educated users rather than the whole world.

The results from the two studies were consistent. In the following sections, we discuss the results of the two studies together, organizing the discussion by the main findings. For brevity, the first study is referred to as the "survey study" and the people who filled out the survey as "respondents", and the second as the "diary study" and the people who took part in it as "participants".

\section{HOW ELECTRONIC AND PAPER TOOLS FOR PIM ARE USED TODAY}

\subsection{Gentlemen prefer electronic while ladies prefer paper-based PIM}

In the survey study, paper-based to-dos were still slightly more commonly used than electronic ones: $70 \%$ of the respondents claimed to use paper todos either All the time or Occasionally while only $50 \%$ claimed to use electronic to-dos All the time or Occasionally. The dominance of paper showed also in the fact that while only $30 \%$ of the respondents used paper to-dos Rarely or Never, $48 \%$ claimed the same for electronic ones. Nevertheless, mixed use was very common: $70 \%$ claimed to use both types of to-dos at least Rarely while only $28 \%$ used only one type (23\% for paper and $5 \%$ for electronic). One respondent claimed never to use either type. Table 1 summarizes the paper and electronic to-do use among the respondents.

Table 1: Use of paper (p) and electronic (e) to-do tools

\begin{tabular}{|r|c|c|c|c|c|}
\hline & $\begin{array}{c}\text { All the } \\
\text { time } \\
(p)\end{array}$ & $\begin{array}{c}\text { Occasionally } \\
(p)\end{array}$ & $\begin{array}{c}\text { Rarely } \\
(p)\end{array}$ & $\begin{array}{c}\text { Never } \\
(p)\end{array}$ & $\begin{array}{c}\text { Total } \\
(e)\end{array}$ \\
\hline All the time (e) & 4 & 4 & 1 & 2 & 11 \\
\hline Occasionally (e) & 5 & 6 & 8 & 1 & 20 \\
\hline Rarely (e) & 7 & 5 & 3 & 0 & 15 \\
\hline Never (e) & 4 & 8 & 2 & 1 & 15 \\
\hline Total (p) & 20 & 23 & 14 & 4 & 61 \\
\hline
\end{tabular}

However, when we compared the paper vs. electronic to-do use between female and male respondents, a different picture emerged. Figures 1 and 2 show the usage frequencies of electronic and paper-based PIM by female and male respondents.

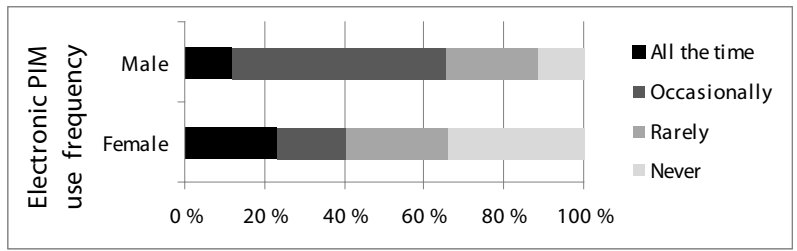

Figure 1: Use of electronic PIM by gender.

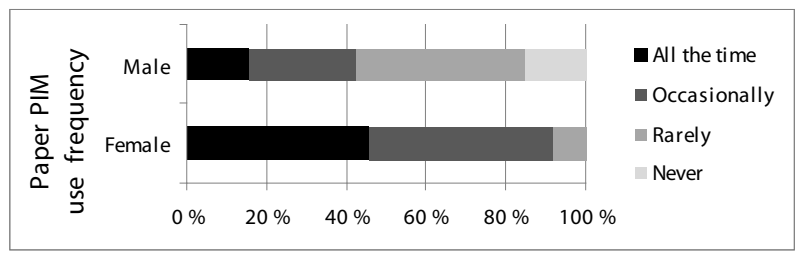

Figure 2: Use of paper-based PIM by gender.

Figure 1 indicates that female respondents were more polarized in their use: There were more really active electronic to-do users as well as those who never used them than in the male group. However, Wilcoxon's two-sample test did not show a statistically significant difference in the distributions. In the case of paper to-dos, however, the difference was highly significant according to Wilcoxon's twosample test $(p<.001)$. As Figure 2 shows, female respondents were more likely to use paper todos and to use them more frequently than male respondents. In fact, all females used paper to-dos while $15 \%$ of the males did not. In contrast, $25 \%$ of the females claimed Never to use electronic todos while only $12 \%$ of the males made the same claim. Overall, however, mixed use was the rule, as $65 \%$ of the female respondents and $77 \%$ of the male respondents used both types of to-dos at least Rarely.

Thus, although the survey study results as whole confirmed earlier reports that paper to-dos are more commonly used than electronic to-dos, the data suggests that while female users still widely use 
paper to-dos, male users use more electronic than paper to-dos. This difference between the genders has not been reported earlier.

The same gender difference also surfaced in the diary study, as all five male smartphone users used electronic calendars-three exclusively so-but two of the six females used only paper calendars. Two used electronic calendars exclusively. Thus, while the smartphone users we interviewed had as a group moved to electronic PIM, the move was more pronounced among the males.

This gender difference might mirror the general trend of males typically adopting new technologies and gadgets first: "The thesis here is that men do not use technology more than women but simply sooner. They are, in diffusion terms, early adopters, but women always catch up" [7]. Thus, the gender difference is not likely to be caused by PIM per se. However, our data does not allow us to hypothesize reasons for the gender difference, and so studying the reasons behind it is left for later work.

\subsection{Survey study: How paper to-dos are used}

The 20 respondents who claimed to use paper todos All the time organized both their everyday and working lives extensively with them. Many prepared a list to organize their activities within a certain timeframe, typically one day or one week: "I tend to start my week by making an updated to-do list where I often plan what to get done each day of the week. The list gets updated and annotated throughout the week." Two even mentioned planning the time necessary for the tasks.

In contrast, the respondents who used paper to-dos Occasionally (23) or Rarely (14) typically used them for special situations, such as preparing for trips or special events, and when they began to feel unable to remember everything that needed to be done. That feeling of losing control was repeatedly mentioned as a trigger to start making to-dos: "Mostly I use them just to clear my mind if I have so many things to do that I can't manage all in my head."

There was great variance in how detailed the to-do item descriptions in the lists were. Some respondents used only keywords to jog the memory while others wrote detailed descriptions and even broke tasks into subtasks. The majority, however, fell somewhere between these two extremes, although leaning towards brevity. Bullet-style was often used, and quite a few respondents mentioned organizing the items in sequential order (the order in which they expect to do the tasks) or in order of importance/ priority. Ordering items by locations where the tasks were to be done was not mentioned (cf. [19]). However, some divided items inside one list by such mental locations as "work" vs. "home" or used separate lists for them.

Striking over the done items was typical. However, the done items were also important to respondents. Checking through the done items provided them the peace of mind that everything was $\mathrm{OK}$ in addition to helping them see what to do next and how to schedule things. Paper to-do items were often annotated with exclamation marks or by using bigger letters to mark the most important items.

We categorized the to-do topics mentioned in the responses into important matters and mundane matters, with deadlines and appointments at work and exams at school categorized as important and shopping lists, household chores, and notes about weapons in Final Fantasy as mundane. The division was based on consequences: Forgetting an important matter can have big consequences while forgetting a mundane matter is not that big a deal. In essence, paper calendars were typically used for important and time-related matters while paper slips were more typically used for mundane and non-timerelated matters. While not all the tasks that ended up on paper slips were unimportant, the tasks that ended up on in calendars were typically important.

Post-its were used widely for both mundane and important matters, as they afforded opportunistic reminding. In fact, many to-dos on paper-slips also ended up being used like post-its with respondents sticking them around for opportunistic reminding. Even calendars were used in a similar fashion: "Usually I keep the calendar open at my desk". This potential for opportunistic reminding is one of the strengths of paper-based to-dos.

Paper slips, calendars, and post-its were the most common tools for paper to-dos, representing the vast majority of the tools used.

\subsection{How mobiles are changing the game}

\subsubsection{To-dos and mobile phones}

While in the survey study we were not directly probing the connection between using electronic to-dos and using mobile phones or other portable devices for to-dos, such connection was noticeable in the electronic to-do use descriptions. In fact, $60 \%$ of the respondents (27 persons) who used electronic to-dos (46 persons) also mentioned using mobile devices, mainly mobile phones ( 25 persons out of 27-in addition one used an iPod and one a PDA), for to-dos. As we did not specifically ask about mobiles but electronic to-do use in general, the actual number is likely to be higher.

In fact, the more the respondent used electronic to- 
dos, the more likely they were to mention using a mobile device: $73 \%$ of those who used electronic todos All the time, $60 \%$ of those who used electronic to-dos Occasionally, and $46 \%$ of those who used electronic devices Rarely mentioned using a mobile device for to-dos.

Consequently, it seems likely that the use of mobiles for to-dos has helped edge electronic to-do use up. Our findings here superficially conflict with earlier studies (e.g. [9, 21]), but when we look at the bigger picture, the conflict turns out to be only apparent. First, people who used mobiles for to-dos often also used paper to-dos widely. Thus, people who constantly use to-dos use many different means, including today increasingly mobile devices. Second, mobile devices, especially phones, have developed after these studies to better support PIM, and so, what was the norm 4-5 years ago does not apply directly anymore.

\subsubsection{Electronic calendars are replacing paper ones} Among the diary study participants, electronic PIM is clearly replacing paper-based PIM. The first casualty has been the paper calendar, as electronic calendars were more popular among our 11 participants: 5 had given up paper calendar altogether, 4 used both (of whom 2 mention edging towards using only electronic calendars), and only 2 used only a paper calendar. Two, both males, used online calendars with mobiles (Google Calendar and Exchange) while other electronic calendar users used the calendar available in their phone. The two paper calendar users did use mobile phone reminders for important or out of ordinary matters.

\subsubsection{Mobile web browsing}

Any device that aims to become central to PIM has to offer Internet connectivity because of the importance of email in PIM. Nine of our smartphone users browsed the web with their phones, either with flat-fee plans or with free WLAN connections. The participants without flat-fee plans used their phones typically for checking specific information, such as opening times or timetables, and occasionally checked their emails, especially if a free WLAN was available. The four users with flat-fee plans, on the other hand, browsed the web more like they did with PCs. Three also used social media with their mobile phones (Facebook and Twitter). Email figured high in their Internet use, too.

However, when it came to entering larger amounts of text than short email replies or status updates in social media, the flat-fee plan owners also preferred to use PC. It was the same for buying things online: The participants did not feel comfortable doing it with the mobile. Better text entry, bigger screen, and better browsers offered by PCs meant that serious Internet use still took place in that domain.
In contrast, mobile browsing took place when having some extra time and not having a PC available, for instance in buses or between classes: "It's just that when I'm not at home or somewhere where there's a computer or laptop available and I have extra time, that's when I use the phone."

\section{DRIVERS OF CHANGE}

\subsection{Penetration and development of mobiles}

One reason why mobile devices are increasingly used for PIM is clearly that they are the electronic device that is always on and always with us: "because the phone is always with me." The survey study showed that the use practices for paper and electronic todos are not that different. Today we more often have our mobiles with us than pen and paper, and with mobiles, we also have our PIM information with us everywhere, either inside the mobile or through Internet connectivity, without us having to remember to print it out or take the relevant paper slip with us.

In fact, convenience was one factor that diary study participants emphasized widely. All participants had their mobiles with them all the time-only exceptions were when doing sports, such as swimming or jogging - while paper calendars were not even for those users who used only the paper calendar. Two participants mentioned having made the switch to the electronic calendar because using both calendars had become too much of a hassle, and the same reason was also evident for the two mixed users who were edging towards using only electronic calendars.

However, the most important reason-and the reason behind the previous one by enabling the choice-appeared to be the advances in the mobile phones. The participants brought up three main reasons why mobiles were increasingly central to PIM: 1) the screens were big enough with good enough resolution, 2) entering information was convenient enough, and 3) accessing Internet with flat-fee plans or free WLAN was practical enough.

Three participants mentioned the bigger screen as a criterion for buying their current phone and one participant who only used paper calendar mentioned that her phone-which had a $128 \times 160$ pixel screen, the smallest in this study-should have a bigger screen for her to use its calendar. Bigger screens were also vital for improved web browsing and document viewing and editing.

Text entry with mobile devices has traditionally been cumbersome $[1,3,4,9]$ but the QWERTY keyboards, increasingly common either as touchscreen solutions or as physical keyboards, 
have helped mobiles to become genuine PIM tools. One participant encapsulated this by stating that the moment he got a phone with QWERTY, he switched to using the phone's calendar.

In addition, three participants mentioned WLAN support being an important criterion in selecting their phones. Internet connectivity was an important part of phones becoming central to PIM, as it enabled both connecting to online communities-Twitter and Facebook by three participants-and, more importantly, made checking emails possible. The centrality of email in PIM $[2,22]$ and in connecting to others means that a mobile PIM device needs to allow using it. While the participants who used their email with the phone typically did not write long messages with it, they liked being able to check their mails freely and to make short replies.

In contrast, not a single participant mentioned improvements in applications and most were content to use catch-all applications or co-opt features for PIM. For instance, two participants mentioned using meetings for all kinds of matters simply because they showed on the idle screen and it was easy to add reminders to them. In fact, having items visible on the idle screen was an important factor in selecting a program to use for many.

The same phenomenon was evident also in the comments by the survey study respondents. When things ended up in the mobile, users often opted for catch-alls and co-opting applications instead of using dedicated applications.

Another tendency was to use whatever was familiar. For example, one diary study participant said that she used SMS message drafts for writing things down in her mobile and that she did not know if there even was another way. Some survey study participants also mentioned using SMS drafts: "I use them if I'm somewhere where I need to quickly take something down..."

In hindsight, it seems obvious that a mobile device with Internet connectivity, big enough screen, and support for convenient text entry for short amounts of text was needed for electronic PIM to start being used widely. In addition to being always with us-"I carry [the phone] with me but I don't carry a computer with me"-the mobile phone also offers other advantages over computers when it comes to PIM: They are always on and the start-up times of applications are very short, so short in fact that one smartphone user mentioned that the time needed to locate either pen and paper or the mobile was a significant factor in deciding which to use: If his calendar was easily reachable, he would use it but if it was at the bottom of his bag, he would then use his mobile phone. Thus, even when sitting at home, checking something quickly from the net or glancing at emails can be more convenient with an alwayson device, and in fact, this is where most mobile browsing has been found to take place $[14,16]$.

\subsection{Reminders: Ace in the hole for mobiles}

\subsubsection{Survey study: Electronic vs. paper reminders} In the survey study, reminding was very common practice with both electronic and paper to-dos. The difference between the two is that while paper reminders are passive (remind by existing in space and waiting to be noticed), electronic reminders can be active (can draw attention to themselves by sound or vibration). Respondents typically used Post-its and other paper reminders in the environment or between calendars-or wrote lists directly on the calendar-to create circumstances where they could opportunistically stumble on the to-dos where and when they needed to act upon them.

While electronic reminders cannot easily be stumbled on in the physical environment, similar strategies were used in virtual environments (inside devices and PCs): To-do files or gadgets were placed on the PC's or phone's desktop to keep them visible and make it possible to glance at them often: "My phone shows a few forthcoming tasks and calendar events on the front view of my cell, so every time I open my phone I tend to quickly take a look if anything urgent is coming up."

In all, eleven survey respondents brought electronic reminders up in their to-do practice descriptions. Reminders were used for a wide range of things, for example "...every evening the mobile rings to make sure I take my birth control pills :-)" and "I set the alarm of the calendar to remind me of deadlines, meetings, etc." Furthermore, the respondents evaluated being able to have time-based reminders as the second most important feature among the 67 features we asked them to rate. Reminders were typically added with calendar applications, especially those in mobiles.

Interestingly, also respondents who otherwise preferred paper to-dos made use of the active reminders: "[My] current system consists of paper calendar and to-dos on paper pieces completed with the reminding functionality in the mobile phone."

There were also requests for automated reminders in the comments, many centering on the need to make setting them easy: "Again, automated deadlines and reminders would be less of a hassle to manage" and "Deadline could come with an automatic reminder of it." Others were not as sure and expressed concerns: 
"It depends on how it reminds me. I don't want any irritating spam or popups" and "Automatic alarms no thanks." Clearly, users want to be in position of power when it comes to automated reminders-or other automated features. Empowering users to be able to suit the device to their ways and not forcing them to adjust to the tyranny of the device is naturally enough the key here: "The speed of usage is of high value for to-dos, thus I like to keep things as simple/ optimal for me as possible."

\subsubsection{Diary study: Mobiles for reminding}

Reminding was the feature that diary study participants uniformly loved and extensively used. Active reminding was seen as superior to the passive reminding allowed by paper: “...l don't always remember to look at my paper calendar, and the mobile phone, it has a reminder with an alarm, that's how I remember it." Nine participants-the ones that used electronic calendars-used reminding all the time. The other 2 who used only paper calendars nevertheless also used mobile phone reminders for special situations and matters. Reminding was not only used to remember things but also to have a peace of mind: A set reminder allowed the participant not to worry about the matter.

Again, reminding can be this popular because it is by a device that we always carry with us and that is always on. It also seems apparent that having reminders available for to-dos and calendars increases the likelihood of things ending up in the mobile in the first place. With our diary study participants, anything that required a reminder ended up in the mobile phone, even if originally noted down on paper.

Nevertheless, passive reminding was also used with mobiles. Many participants mentioned making sure that important things were visible on the idle screen of their mobiles so that every time they glanced at their mobiles, they were reminded of things to do. As mentioned, co-opting features was used to accomplish this: "If I have something I need to take care of, for instance I need to pick up keys from some place, then the meeting marking shows up on the idle screen. So, when I look at the phone, I see the 'meetings', what I have today, no matter what they are as long as they are marked to be meetings. ... If I used tasks, I'd be limited to three or something like that [on the idle screen]." One participant even said that if he could not have things visible/accessible on the idle screen, he would rather use paper than look for such things as shopping lists from inside the phone.

The benefits of active reminding are such that no PIM application should be without them, as our diary study indicated. In addition, the smartphone user experiences emphasize that it is of utmost importance to make sure that both active and passive reminding is easy for all PIM applications. The importance of reminders was speculated by Colbert [8], and our study confirms his hypotheses.

\section{PAPER VS. ELECTRONIC PIM IN THE FUTURE: ISSUES TO APPRECIATE}

\subsection{Context and content decide the medium}

In the Related work section we already discussed various reasons for the advantages that paper-based PIM tools offer. However, it has not been purely the strengths that paper has that have tipped the scale in its favor. As mobile phones have been common already for some time now, it appears that the relative weaknesses of them as PIM tools have played a part in the equation, too. As mentioned, the most blatant ones appear to have been poor inputting and small screens. As these have improved, mobiles have become real contenders to paper.

The use of SMS drafts for short notes while better tools, such as Notes, exist also suggests that not all users have found the tools available in their phones. Perhaps users simply continue to use the old ways because that is what they know and what has worked for them. As the new generations use mobiles from very young age on, they are likely to be more familiar with all the possibilities offered by mobiles and to use them.

PCs were unable to challenge paper partly because they were not portable and not always on. In any case, without telephony and Internet, we might not be as likely to carry mobile computing devices everywhere with us even today. Before mobiles, it was paper that was ubiquitously available, and so, before improved inputting and bigger screens of the mobiles reached the tipping point, paper continued to be the medium of choice.

All this is not to say that there will be no use for paper in PIM in the future. In the diary study, all but one participant used both paper and electronic to-dos: All used paper to-dos and only one did not use electronic to-dos. While there are no absolutes in where to-dos and short notes ended up-for instance, availability affected the equation-there were some clear tendencies evident. One observation was that at home or work, if the content was to be used in that location, there was a tendency to write it on paper (or occasionally in a file in the computer if the work was to be done with the computer) but if the content was to be used outside, it tended to end up in the mobile. Furthermore, general musings tended to end up on paper while content that was needed long-term or permanently tended to end up in the mobile, as 
paper was seen as impermanent and easily losable. Moreover, important and specific matters tended to end up in the mobile. Shorter lists and individual items also had a tendency to end up in the mobile while longer lists more easily ended up on paper.

\subsection{To compete with paper, be like paper}

When we asked the smartphone users what it would take for them to give up on paper altogether in PIM, the answer was almost unanimous: $A$ portable phone/device that would allow paper-like interaction and most of the things that paper allows. Five participants mentioned being able to write on the device like on paper as important, and 2 more emphasized easiness of input without mentioning writing directly on the device.

Paper-like input did not only refer to being able to write directly on the device, as two participants also mentioned wanting to be able to embellish the texts with drawings and markings for indicating relationships and such between and within the entries.

Furthermore, the screen should be big enough to view wholes, such as one week's calendar, comfortably. Screen size, however, is a two-edged sword, as two participants mentioned that Nokia's Communicator was already a bit too big for comfort.

Based on our data, mixed use is the order of today. However, the point where electronic PIM is more common that paper-based PIM also for Joe Blow appears to be inching closer. The mobile phone seems the most likely candidate for becoming the central device in electronic PIM, being the device that we have with us all the time-_because it's always with me"-and being always on. In any case, the device to take the central place in PIM also needs to offer Internet connectivity and telephony or we would have to carry two devices with us and that seems less likely to happen.

\subsection{Sharing in PIM: A non-issue}

A particular motivation for our survey study was to gain input for designing a shared to-do widget. However, sharing electronic to-dos hardly came up in use descriptions in the survey study. In fact, only one respondent mentioned sharing, and even there the document in question was a requirements specification for designing a new application at work. Also, the top ten most important features according to the respondent ratings had nothing to do with sharing while four among the ten least important features dealt directly with sharing. Clearly, electronic to-dos were seen as personal tools rather than as tools for collaboration.
This is interesting as sharing is often seen as an advantage that electronic to-dos have over paper. The diary study shed some light on the reasons behind this: Most participants simply did not find sharing to-dos or calendars pertinent to their lives. While they were not against sharing per se, most of them did not see much need for it in their own lives, either: "...l can't think of anything where l'd need a shared to-do list. ...if somebody showed me a working system, a useful system, I don't think I'd oppose it or anything. I just haven't missed one this far."

While participants did not have much experience of to-do or calendar sharing, some nevertheless had negative experiences. Most complaints centered on users rather than the applications: "It [Google Docs used in a student project] hasn't worked quite as we wished but it's perhaps not the tool's fault-it's more that we haven't managed to organize ourselves to using it yet."

Only one participant had had a positive experience of to-do sharing, and that was related to a tasksharing tool for Scrum at work. Also, two participants had positive experiences of with-family sharing: One participant shared his Google Calendar with his spouse and another participant used Google Latitude with her spouse. However, although some other participants were also married or lived in a relationship, they had not been interested in sharing their electronic PIM information with their partners.

In fact, only the participant who had shared his calendar with his spouse was enthusiastic about sharing calendars and to-dos in general: "...if it were up to me, electronic calendar system is a brilliant thing ... it would ease things no end" even though he also had had negative experiences of them, again due to users: "At work we had personnel's club and I set up an [electronic] calendar for us ... The only problem was that nobody else knew how to use it ... I would've been the only user."

All in all, smartphone users in general did not see the relevance of sharing in PIM for themselves although they were not against idea as such. The main problem according to their experience were not the applications but other humans, who could defeat the best-designed systems with laziness and indifference.

\section{CONCLUSIONS}

Our two studies suggest that electronic PIM is poised to become more common than paper-based PIM in near future, as both indicate that the use of electronic PIM has become much more commonplace than 
previously reported. Already today mixed use is the most common approach according to the survey study, and among the lead users, electronic calendars were used more commonly than paper calendars. Interestingly, mobile phones appear to be behind this development. In the diary study, mobiles had already become the epicenter of electronic PIM, and in the survey study, the more the respondents used electronic to-dos, the more likely they were to use mobile phones in PIM.

In hindsight, it is easy to see why mobile phones were a precondition for electronic PIM to really catch on. After all, PIM is all "about external artifacts that augment human cognition" [11] and thus these artifacts need to be available to us when and where we need them. PCs, not even mini laptops of today, are not always with us and they are not always on. Thus, the speed and availability of paper in different situations was not possible to beat in the past. Now, however, we carry mobiles with us all the time and everywhere and they are always on: "I write things down as they come to mind: works fine, because the phone is always with me." This makes it convenient to use them both for entering and storing information: They are there when we need to write down something and when we need to access some information.

Furthermore, mobile phones allow us to use active reminders. While reminding has all along been part of PIM with paper notes and such placed in the environment for passive reminding, mobile phones with active reminders have become the norm when we need to remember things, especially when on the move. Although active reminders have been possible with PCs for some time now and are still used, it is the mobile phone that is always on and always with us, and so it was the mobile phone that was able to offer an advantage that paper simply could not match. While some computer-related work reminding is still in the PC and passive paper reminders are used at home and work, even the participants and respondents who used mainly paper-based PIM tools also often used mobile phone reminders.

However, we have carried mobiles with us for a long time without them really catching on as means to PIM. It was only with better screens and better text entry-especially with touchscreen or physical QWERTY keyboards-that the more developed phones of today offer that mobiles began to be more widely used for PIM. Another contributing factor is faster and cheaper Internet connectivity that allows email to move with us everywhere.

Consequently, with to-dos, calendars, address books, and emails all in mobile phones, it is easy to see why one smartphone study participant stated that "...because it's always with me, I can organize my life immediately and know what's going on." In effect, mobile phones were already the epicenters of PIM for smartphone users and likely to become that for the rest of us in the not-so-distant future. A factor that may accelerate this development is the increasing popularity of social network sites. While it is often sufficient just to browse incoming emails, social networking is most rewarding for users that participate actively, thus making entering information with a mobile device salient. For instance, today more than 100 million "active users" access Facebook through their mobile devices, and these users are twice as active on Facebook as non-mobile users [10]. For these users, the versatile use of mobile PIM tools is natural.

This means that when designing PIM applications, taking mobile phones into consideration is a makeor-break factor. PIM is becoming mobile and PIM applications have to support this. However, as mobile phones are still only convenient enough to input small amounts of information and not that convenient for organizing it, PCs still have an role to play in PIM (in addition to being central to PIM related to the work that is done with a PC in the first place-a to-do list for work that is to be done with a PC is still likely to end up in the PC, and this is unlikely to change).

With Internet connectivity in mobiles becoming more of a rule than exception-according to PEW report, in the US " $83 \%$ of adults have cell phones or smartphones and, among them, $35 \%$ have accessed the internet via their phone" [17] -it seems likely that PIM information will increasingly be available on the Internet and we just access it with different devices, such as mobile phones (and other mobile devices) and game consoles in addition to PCs: "I have a lot of different devices and workplaces in my use so I have to be able to read my [to-do] lists everywhere" (survey respondent). Also, even the smartphone users in the diary study seldom synchronized the data in their mobiles with the data in their PCs, meaning that synchronization has to be automatic or the same data available for all devices on the Internet. The different devices will need different interfaces that support the typical use cases for the device. Thus, when designing PIM applications, the typical use and use context of the device need to be taken into consideration.

\section{ACKNOWLEDGMENTS}

This work was supported by the ICT SHOK project on Flexible Services. The third author was supported by the Academy of Finland grant 1130044 while the author was visiting the University of Canterbury in Christchurch, New Zealand. 


\section{REFERENCES}

[1] Bellotti, V., Dalal, B., Good, N., Flynn, P., Bobrow, D. G., and Ducheneaut, N. (2004) What a To-do: Studies of Task Management towards the Design of a Personal Task List Manager. CHI '04, Vienna, Austria, April 24-29, 735-742. ACM Press, New York, NY.

[2] Bellotti, V., Ducheneaut, N., Howard, M., Smith, I., and Grinter, R. E. (2005) Quality Versus Quantity: E-mail-centric Task Management and Its Relation with Overload. Human-Computer Interaction, 20 1-2, 89-138.

[3] Bellotti, V. and Smith, I. (2000) Informing the Design of an Information Management System with Iterative Fieldwork. DIS '00, New York City, NY, USA, Aug. 17-19, 227-237. ACM Press, New York, NY.

[4] Bernstein, M., Van Kleek, M., Karger, D., and Schraefel, M. C. (2008) Information Scraps: How and Why Information Eludes Our Personal Information Management Tools. TOIS, 26 4, 1-46.

[5] Blandford, A. E. and Green, T. R. G. (2001) Group and Individual Time Management Tools: What You Get is Not What You Need. Personal and Ubiquitous Computing, 5 4, 213-230.

[6] Brush, A. J. B. and Turner, T. C. (2005) A Survey of Personal and Household Scheduling. GROUP' 05 , Sanibel Island, FL, USA, 330-331. ACM Press, New York, NY.

[7] Brynin, M., Raban, Y., and Soffer, T. (2000) The New ICTs: Age, Gender and the Family. Chapter 5 of the book manuscript by the EU FP5 project "e-Living - Life in a Digital Europe". http://archive.eurescom. eu/e-living/deliverables/e-liv-D14-Ch5-Family.pdf (March 18, 2010)

[8] Colbert, M. (2005) Age Differences Rendezvousing: Reminders for Side-stepping. Personal and Ubiquitous Computing, 9, 404-412.

[9] Dai, L., Lutters, W. G., and Bower, C. (2005) Why Use Memo for All? Restructuring Mobile Applications to Support Informal Note Taking. CHI EA '05, Portland, OR, USA, April 2-7, 1320-1323. ACM Press, New York, NY.

[10] Facebook Statistics (2010) http://www.facebook. com/press/info.php?statistics (March 18, 2010)

[11] Gil, Y. and Ratnakar, V. (2008) Towards Intelligent Assistance for To-do Lists. IUI '08, Gran Canaria, Spain, February 14-17, 329-332. ACM Press, New York, NY.
[12] Hippel, E. von. (2005) Democratizing Innovation. The MIT Press, Cambridge, MA.

[13] Jones, W. and Teevan, J. (eds.) (2007) Personal Information Management. University of Washington Press, Seattle, WA.

[14] Kaikkonen, A. (2008) Full or Tailored Mobile Web- Where and How Do People Browse on Their Mobiles? Mobility '08, Yilan, Taiwan, Sept. 10-12, 1-8. ACM Press, New York, NY.

[15] Lin, M., Lutters, W. G., and Kim, T. S. (2004) Understanding the Micronote Lifecycle: Improving Mobile Support for Informal Note Taking. $\mathrm{CHI}$ '04, Vienna, Austria, April 24-29, 687-694. ACM Press, New York, NY.

[16] Nylander, S., Lundquist, T., and Brännström, A. (2009) At Home and with Computer Access - Why and Where People Use Cell Phones to Access the Internet. $\mathrm{CHI}$ '09, Boston, MA, USA, April 04-09, 1639-1642. ACM Press, New York, NY.

[17] Rainie, L. (2010) Internet, Broadband, and Cell Phone Statistics. Pew Internet \& American Life Project: PewResearchCenter, Washington, DC.

[18] Reimer, Y. J., Brimhall, E., Chen C., and O'Reilly, K. (2009) Empirical User Studies Inform the Design of an E-notetaking and Information Assimilation System for Students in Higher Education. Computers \& Education, 52 4, 893-913.

[19] Taylor, A. S. and Swan, L. 2004. List Making in the Home. CSCW '04, Chicago, IL, USA, Nov. 6-10, 542-545. ACM Press, New York, NY.

[20] Teevan, J., Jones, W., and Bederson, B. B. (2006) Introduction to Special Issue on Personal Information Management. CACM, 49 1, 40-43.

[21] Tomitsch, M., Grechenig, T. and Wascher, P. (2006) Personal and Private Calendar Interfaces Support Private Patterns: Diaries, Relations, Emotional Expressions. NordiCHI'06, Oslo, Norway, Oct. 14-18, 401-404. ACM Press, New York, NY.

[22] Whittaker, S. and Sidner, C. (1996) Email Overload: Exploring Personal Information Management of Email. CHI '96, Vancouver, Canada, April 13-18, 276-283. ACM Press, New York, NY. 